Classification

Physics Abstracts

$61.70 \mathrm{~W}-61.80 \mathrm{~B}-73.30$

\title{
Caractéristiques électriques de diodes Au-Si(N) réalisées après irradiation par laser
}

\author{
J. P. Ponpon, E. Buttung et P. Siffert \\ Groupe de Physique et Applications des Semiconducteurs, (Phase) \\ Centre de Recherches Nucléaires, 67037 Strasbourg Cedex, France
}

(Reçu le 25 mars 1982, révisé le 17 mai 1982, accepté le 25 mai 1982)

\begin{abstract}
Résumé. - On a préparé des diodes Schottky par dépôt d'une électrode d'or sur du silicium de type $\mathrm{N}$ dont la surface avait préalablement été irradiée par une impulsion lumineuse brève issue d'un laser à rubis. L'augmentation de l'énergie des impulsions au-delà d'un seuil situé aux environs de 0,7 à $0,8 \mathrm{~J} / \mathrm{cm}^{2}$ provoque une forte dégradation des caractéristiques électriques des diodes, la réduction de la barrière de potentiel au contact or-silicium et l'accroissement du facteur d'idéalité. Les mesures de capacité montrent que le recuit par laser introduit, dans une zone superficielle du silicium, une forte densité de défauts de type donneur dont on a pu, par décapages successifs de la surface, déduire le profil de distribution pour une irradiation effectuée à l'énergie de $1,45 \mathrm{~J} / \mathrm{cm}^{2}$. La guérison de ces défauts par un recuit thermique permet de rétablir en partie les propriétés des contacts or-silicium.
\end{abstract}

\begin{abstract}
Schottky diodes have been realized by deposition of gold contacts on N-type silicon after annealing of the surface by means of a $Q$-switched ruby laser. The electrical characteristics have been measured as a function of the laser energy up to $1.6 \mathrm{~J} / \mathrm{cm}^{2}$. A strong degradation in $I-V$ curves, barrier height and diode quality factor has been observed with increasing energy. In any case, strong change in electrical properties occurs for energies higher

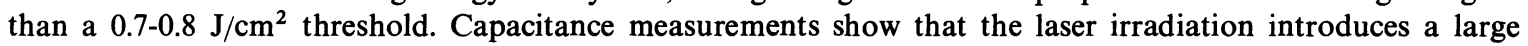
density of donor defects in the surface region. Their distribution profile has been deduced, for a $1.45 \mathrm{~J} / \mathrm{cm}^{2} \mathrm{irra-}$ diation, by using a stripping process and capacitance measurements. The evolution of these properties on thermal annealing also has been investigated.
\end{abstract}

1. Introduction. - Le nettoyage de la surface d'un métal ou du silicium à l'aide de l'impulsion lumineuse issue d'un laser de puissance a été étudié il y a plusieurs années $[1,2]$; dès 1971 , les avantages et les inconvénients inhérents à cette méthode avaient été énumérés [3]. Récemment, le développement de l'utilisation des recuits par impulsions d'énergie a conduit à reconsidérer le problème de l'interaction d'un rayonnement laser avec la surface d'un semiconducteur, d'un point de vue théorique [4] et expérimental [5-9]. Il est ainsi apparu que pour une irradiation effectuée sous ultravide, la concentration d'impuretés à la surface, en particulier de carbonè et d'oxygène, diminuait fortement, conduisant à des surfaces atomiquement propres et que la structure de la surface n'était en outre pas reconstruite, contrairement à ce qui résulte d'un recuit thermique conventionnel; elle serait même assez désordonnée [10]. Le mécanisme qui permet le nettoyage du silicium demeure cependant mal compris : si l'évaporation sous irradiation paraît très probable, la diffusion des impuretés, en particulier de l'oxygène, durant la période où le matériau passe par une phase liquide, est controversée $[9,11,12,13]$. Ce problème est particulièrement important lorsque les irradiations sont effectuées à l'air ou lorsqu'une couche d'oxyde est présente sur les échantillons [14], ce qui est généralement le cas dans les applications pratiques. Par ailleurs, l'irradiation par un laser de puissance provoque l'apparition de défauts, tant dans les métaux [15], que dans le silicium [16] où ils introduisent des niveaux pièges dans la bande interdite $[17,18]$. Leur étude a surtout été effectuée pour des couches de silicium implantées et peu de travaux ont été consacrés à leur caractérisation dans le silicium vierge. Il a cependant été suggéré que des impuretés (oxygène, azote, carbone en particulier) ou des imperfections cristallines, pourraient être à leur origine. De tels défauts étant susceptibles d'affecter le comportement des matériaux, on a cherché dans cet article à préciser l'évolution, en fonction de l'énergie des impulsions provenant d'un laser à rubis, des caractéristiques électriques de diodes Schottky réalisées sur des surfaces de silicium préalablement irradiées. On a ensuite essayé d'en déduire quelques propriétés des défauts liés à l'interaction laser-silicium. 
2. Résultats expérimentaux. -2.1 Préparation DES ÉCHANTILlONS. - On a utilisé du silicium de type $\mathrm{N}$ de résistivité comprise entre 5 et $8 \Omega . \mathrm{cm}$, orienté selon l'axe (111). La plupart des échantillons provenaient de lingots tirés par la méthode de Czochralski (silicium CZ), cependant du matériau préparé par fusion de zone (silicium FZ) a également été employé. Les surfaces étaient de qualité poli optique et nettoyées à l'acide fluorhydrique avant l'irradiation effectuée à l'air à l'aide d'un laser à rubis fonctionnant en mode déclenché et délivrant des impulsions de 25 ns (largeur à mi-puissance), d'énergie comprise entre 0,1 et $1,6 \mathrm{~J} / \mathrm{cm}^{2}$. Le diamètre du faisceau était de $8 \mathrm{~mm}$. Des mesures d'ellipsométrie (sensibles entre autres à la rugosité de la surface ou à la présence d'une couche superficielle amorphe) réalisées à la longueur d'onde $632,8 \mathrm{~nm}$ pour un angle d'incidence de $70^{\circ}$, n'ont pas mis en évidence de différence notable avant et après irradiation, montrant par là qu'aux énergies utilisées la surface du silicium n'était pas très endommagée, à l'échelle macroscopique tout au moins.

Immédiatement après le traitement par laser, les échantillons étaient placés dans une enceinte à vide et un contact d'or de $20 \mathrm{~nm}$ d'épaisseur et de $1 \mathrm{~mm}$ de diamètre (pour les mesures de capacité) ou de $4 \mathrm{~mm}$ (pour les mesures de courant) était déposé au centre de l'impact. Le contact arrière était assuré par une électrode d'aluminium.

2.2 Caractéristiques Courant-tension. - On a représenté sur la figure 1 un exemple de l'évolution des caractéristiques $I-V$ relevées, dans l'obscurité, en fonction de l'énergie d'irradiation pour du silicium FZ.

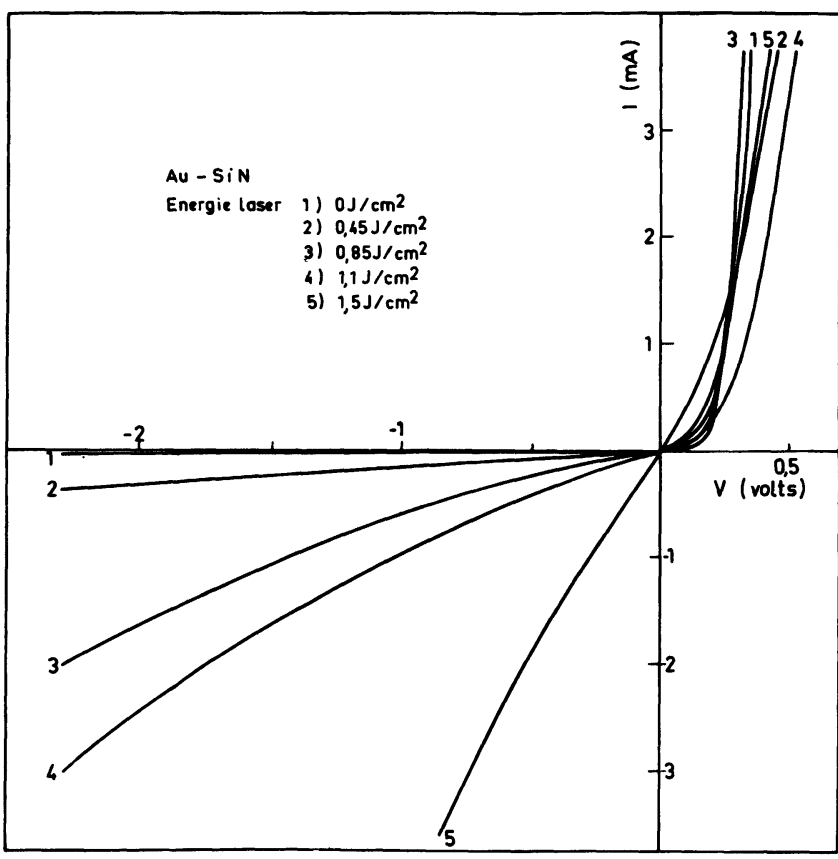

Fig. 1. - Influence de l'énergie d'irradiation de la surface du silicium sur les caractéristiques $I-V$ des diodes $\mathrm{Au}-\mathrm{Si}$.

[Influence of laser energy on the $\mathrm{I}-V$ characteristics of $\mathrm{Au}-\mathrm{Si}$ diodes realized on irradiated Si surfaces.]
La dégradation de ces courbes lorsque l'énergie augmente est évidente, particulièrement pour des énergies supérieures à $0,8 \mathrm{~J} / \mathrm{cm}^{2}$. Au-delà de $1 \mathrm{~J} / \mathrm{cm}^{2}$ le redressement au contact or-silicium $\mathrm{N}$ disparait. Des résultats analogues ont été obtenus pour des substrats $\mathrm{CZ}$.

2.3 Courant De SATURATION ET FaCteur D'idÉaLITÉ DES DIODES. - La figure 2 représente l'évolution, en fonction de l'énergie des impulsions, du courant de saturation $I_{0}$ des échantillons, déduit de la caractéristique $I-V$ aux faibles polarisations directes. Le traite-

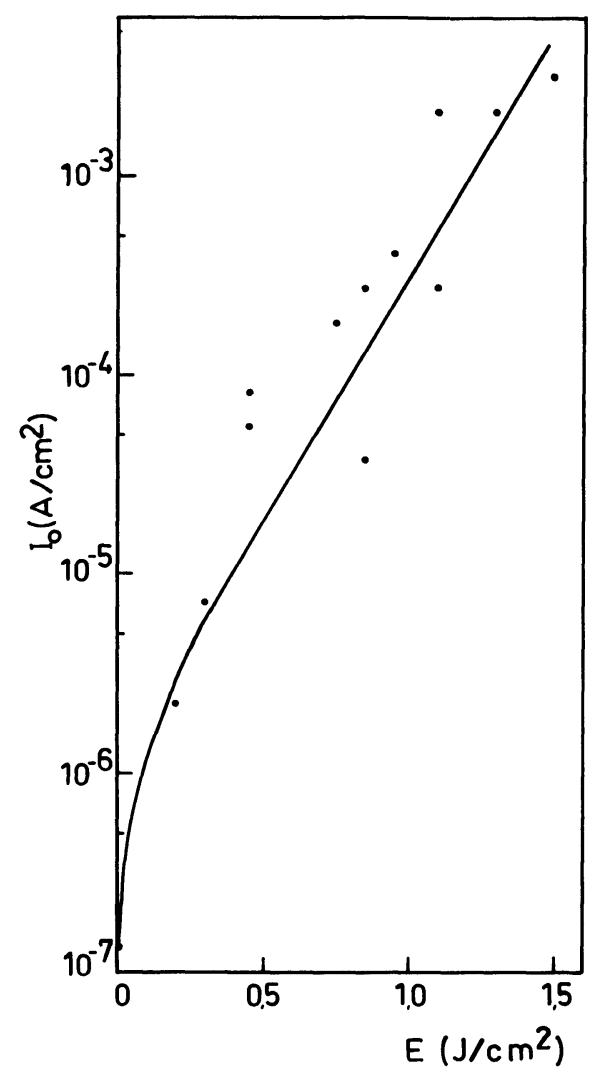

Fig. 2. - Influence de l'énergie d'irradiation de la surface du silicium sur la valeur du courant de saturation au contact $\mathrm{Au}-\mathrm{Si}$

[Influence of laser energy on the saturation current at the Au-Si contact.]

ment par laser provoque, par rapport à un échantillon témoin (non irradié) une forte augmentation de $I_{0}$, qui atteint $10^{4}$ pour une énergie de $1,5 \mathrm{~J} / \mathrm{cm}^{2}$. La hauteur de la barrière de potentiel que l'on peut associer à $I_{0}$ est ainsi réduite d'environ 0,25 à $0,30 \mathrm{eV}$ pour une irradiation à $1,5 \mathrm{~J} / \mathrm{cm}^{2}$ (elle vaut $0.8 \mathrm{eV}$ pour la diode de référence). Conjointement, le facteur d'idéalité $n$ des diodes, qui est de 1,2 pour l'échantillon témoin, augmente de façon notable au-delà de 0,7 à $0,8 \mathrm{~J} / \mathrm{cm}^{2}$ pour atteindre des valeurs très élevées (Fig. 3).

2.4 Mesures De CapaCité. - Des mesures de capacité en fonction de la tension ont été effectuées sur 


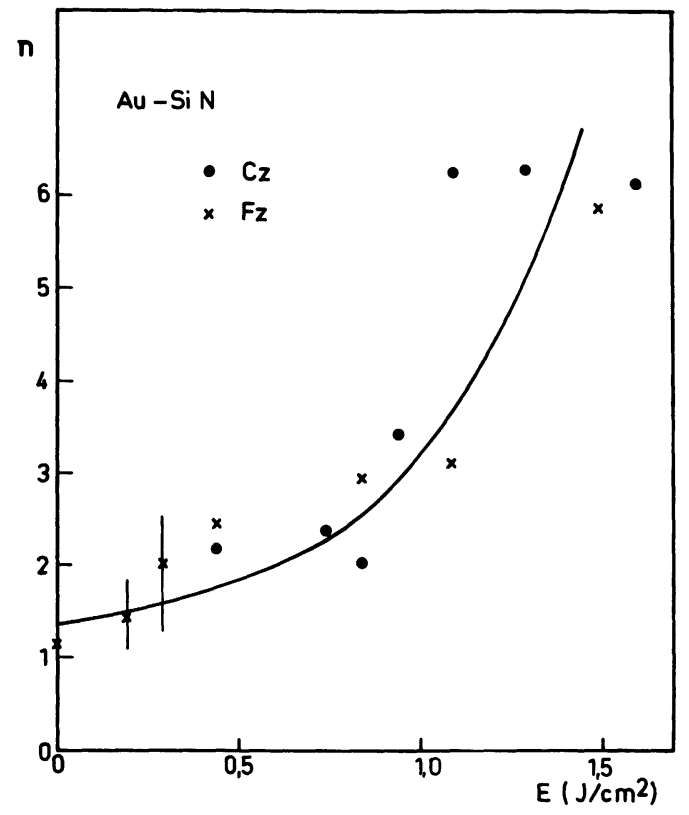

Fig. 3. - Evolution avec l'énergie de l'irradiation du facteur $n$ des diodes Schottky Au-Si.

[Evolution of the diode factor $n$ with laser energy.]

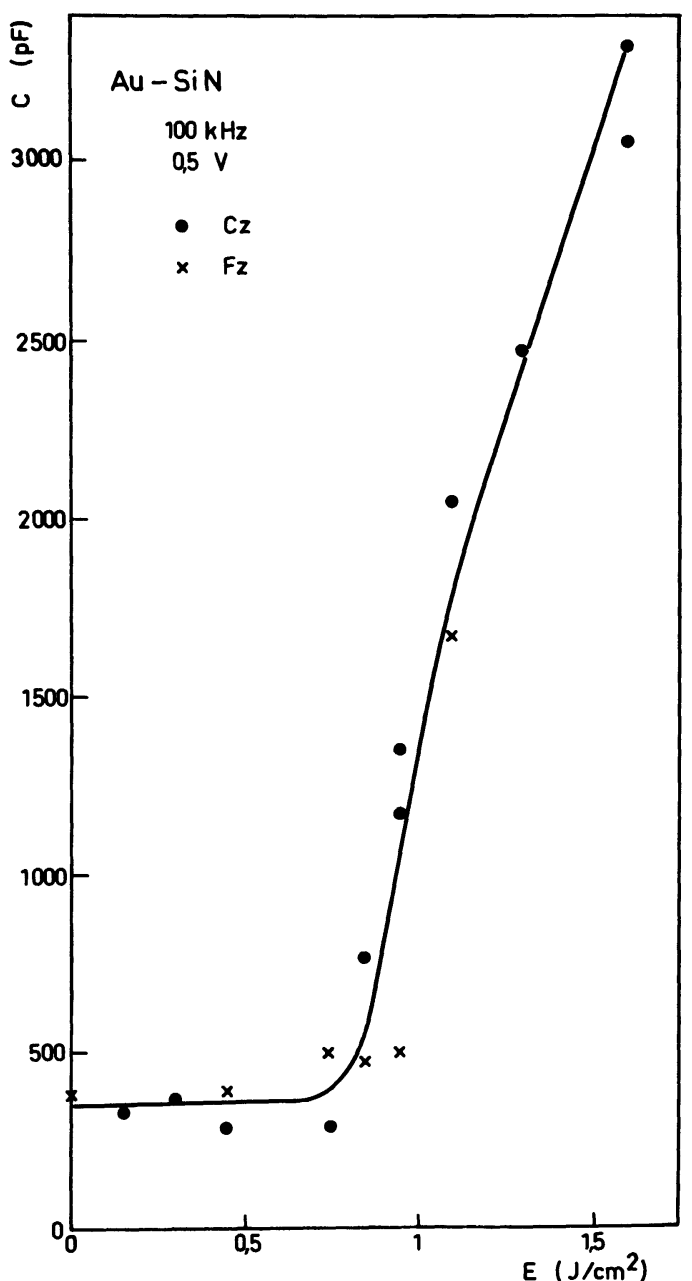

Fig. 4. - Modification de la capacité, mesurée à - 0,5 V, de diodes $\mathrm{Au}-\mathrm{Si}$ après irradiation de la surface du silicium.

[Evolution of the capacitance of Au-Si diodes at $-0.5 \mathrm{~V}$ as a function of laser irradiation.] du silicium $\mathrm{FZ}$ et $\mathrm{CZ}$; aucune différence nette n'est cependant apparue entre ces deux types de matériaux. Du fait du mauvais redressement de certains contacts, nous avons limité les mesures à des tensions faibles. L'évolution de la capacité avec l'énergie pour une tension de polarisation inverse de $0,5 \mathrm{~V}$ et une fréquence de mesure de $100 \mathrm{kHz}$, est représentée sur la figure 4 : jusqu'à un seuil voisin de $0,7 \mathrm{~J} / \mathrm{cm}^{2}$, la capacité des échantillons irradiés demeure très proche de celle de l'échantillon témoin ; au-delà de $0,8 \mathrm{~J} / \mathrm{cm}^{2}$, elle croît fortement et augmente de près de 10 fois pour $1,5 \mathrm{~J} / \mathrm{cm}^{2}$. On a également pu observer sa forte dépendance avec la fréquence du signal de mesure, entre les limites imposées par l'appareillage utilisé, soit de 5 à $500 \mathrm{kHz}$.

2.5 DéCaPages SuCCessifs de la SURFaCe. - Pour un échantillon irradié à $1,45 \mathrm{~J} / \mathrm{cm}^{2}$ on a, par oxydation anodique suivie d'une attaque à l'acide fluorhydrique, successivement enlevé des couches d'environ $12 \mathrm{~nm}$ d'épaisseur. Après chaque attaque, une électrode d'or

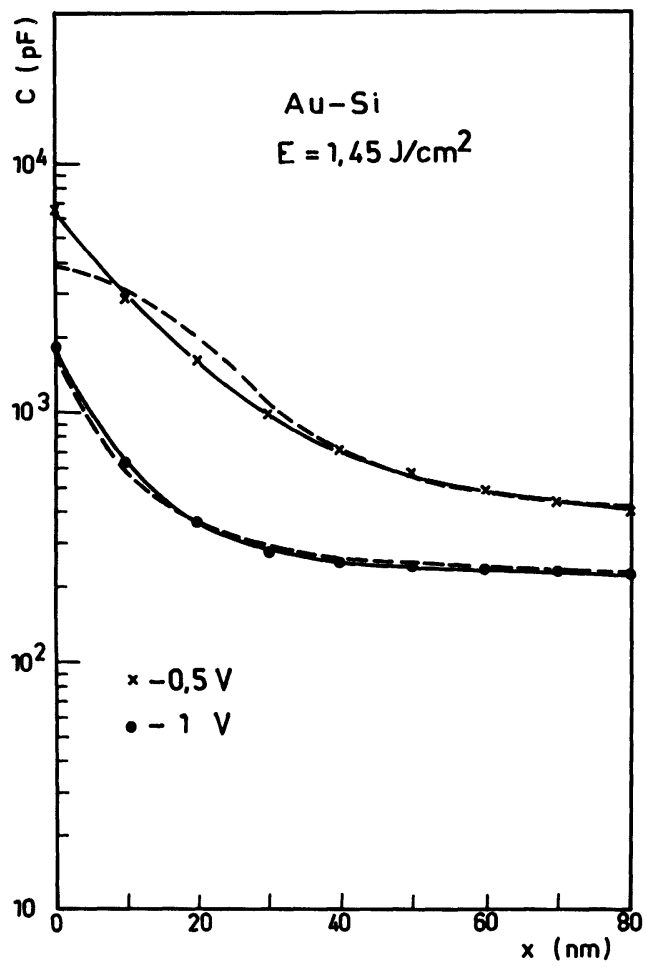

Fig. 5. - Evolution de la capacité pour une diode $\mathrm{Au}-\mathrm{Si}$ réalisée sur un échantillon irradié à $1,45 \mathrm{~J} / \mathrm{cm}^{2}$, en fonction de la profondeur $x$ de silicium enlevée par oxydation anodique. Les courbes expérimentales mesurées à $-0,5$ et - $1 \mathrm{~V}$ (traits pleins), ont été reproduites par le calcul (pointillés) en tenant compte, dans l'équation de Poisson, d'une distribution de donneurs de la forme $N(y)=N_{\mathrm{D}}+$ $N_{\mathrm{T}} \exp (-\alpha y)$.

[Change in capacitance of a $1.45 \mathrm{~J} / \mathrm{cm}^{2}$ irradiated silicon surface as a function of the depth of silicon removed by anodic oxydation. The experimental data at -0.5 and $-1 \mathrm{~V}$ (full lines) have been fitted with calculated curves (dashed lines) obtained by introducing in Poisson's equation the donor distribution $N(y)=N_{\mathrm{D}}+N_{\mathrm{T}} \exp (-\alpha y)$.] 
était redéposée de sorte que l'on a pu mesurer l'évolution des propriétés des diodes en fonction de l'épaisseur de silicium enlevée. La détermination de la hauteur de barrière conduit à des résultats peu significatifs, probablement à cause du mauvais état de surface laissé par le traitement. Le facteur $n$ par contre décroît, après enlèvement d'environ 100 à $120 \mathrm{~nm}$, de 5-6 à 1,8. L'influence du décapage apparaît beaucoup plus nettement sur les caractéristiques $I-V$, en particulier en inverse : pour une profondeur de $100 \mathrm{~nm}$ on observe à $-1 \mathrm{~V}$, par exemple, une réduction du courant inverse de deux ordres de grandeur. Les courbes $I-V$ deviennent alors très proches de celles de l'échantillon témoin. De même, les caractéristiques capacité-tension se modifient notablement avec la profondeur d'attaque : à $-0,5$ et $-1 \mathrm{~V}$ par exemple, la capacité redevient égale à celle de l'échantillon témoin au-delà de $60 \mathrm{~nm}$ environ (Fig. 5). Toutefois, des mesures en fonction de la fréquence font apparaître une dépendance jusqu'à 100-200 $\mathrm{nm}$. On peut également remarquer que le tracé de $1 / \mathrm{C}^{2}$ en fonction de la tension inverse ne devient linéaire que pour des épaisseurs enlevées supérieures à 30-40 nm environ.

2.6 RECUIT THERMIQUE. - On a étudié l'évolution des propriétés des diodes à la suite d'un recuit ther-

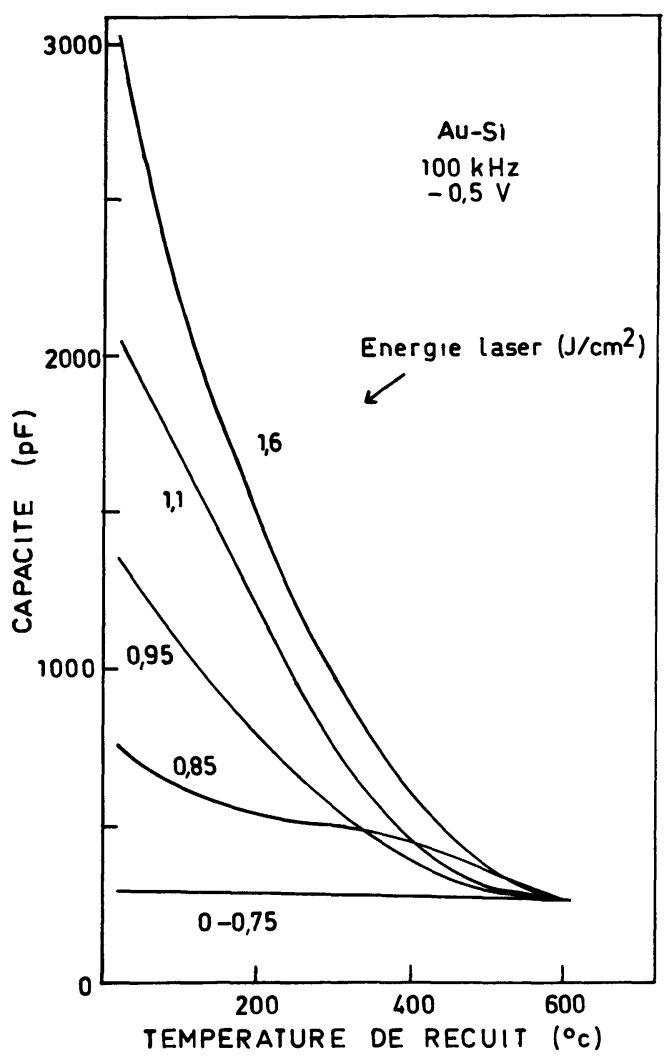

Fig. 6. - Evolution de la capacité de diodes Au-Si réalisées après irradiation de la surface à différentes énergies en fonction d'un recuit thermique de $30 \mathrm{~min}$. sous vide.

[Evolution on thermal annealing ( $30 \mathrm{~min}$. under vacuum) of the capacitance of $\mathrm{Au}-\mathrm{Si}$ diodes for various irradiation energies of the silicon surfaces.] mique, effectué sous vide $\left(p=10^{-6}\right.$ torr $)$ durant $30 \mathrm{~min}$. Les électrodes d'or et d'aluminium étaient otées avant le chauffage et redéposées ensuite. L'augmentation de la température de recuit s'accompagne d'une amélioration des caractéristiques $I-V, C-V$, de la hauteur de barrière et du facteur d'idéalité. Pour des températures de l'ordre de 550 à $600^{\circ} \mathrm{C}$, la capacité des échantillons irradiés rejoint celle de l'échantillon témoin, ainsi qu'il apparaît sur la figure 6 où l'on a représenté, pour différentes énergies d'impulsion, l'évolution de la capacité à - $0,5 \mathrm{~V}$ en fonction de la température de recuit. Toutefois, même après chauffage à $600^{\circ} \mathrm{C}$, les échantillons ne recouvrent pas tout à fait les caractéristiques de la diode témoin : pour une surface irradiée à $1,1 \mathrm{~J} / \mathrm{cm}^{2}$ par exemple, après traitement thermique le courant inverse diminue d'un facteur dix mais demeure encore dix fois plus élevé que celui de la référence; la hauteur de barrière croît de 0,57 à $0,7 \mathrm{eV}$ (au lieu de 0,8 ) et le facteur d'idéalité passe de 3,5 à 1,7 (au lieu de 1,2).

3. Discussion. - Les résultats expérimentaux présentés ci-dessus montrent nettement la forte influence, sur le fonctionnement des diodes Schottky, de l'irradiation par laser de la surface avant le dépôt du métal. On essaiera dans cette partie de déduire de l'étude des caractéristiques électriques de ces diodes quelques propriétés de l'interaction entre une impulsion laser de puissance et le silicium, et de montrer qu'elles peuvent, au moins en partie, résulter de la création de défauts introduisant des niveaux dans la bande interdite lors de l'irradiation.

3. 1 SEUIL D'ÉNERGIE. - L'évolution des caractéristiques courant-tension, de la capacité et du facteur d'idéalité $n$ en fonction de l'énergie montre que jusqu'à $0,7-0,8 \mathrm{~J} / \mathrm{cm}^{2}$ l'influence de l'irradiation par laser sur les propriétés des diodes Schottky demeure faible; au-delà de ce seuil, le changement est notable et s'accompagne de la dégradation des courbes $I-V$ et d'une forte augmentation de $n$ et de la capacité. Le fait qu'un tel seuil n'apparaisse pas nettement sur le comportement du courant de saturation vient probablement d'une modification des propriétés de la surface, même pour des irradiations à faible énergie. On peut noter que le seuil défini par ces expériences correspond au seuil de fusion du silicium calculé en prenant en compte un modèle purement thermique [19]. Dans le cas d'une irradiation par un laser Nd-Yag (durée d'impulsion de $100 \mathrm{~ns}$ ), des mesures analogues ont permis de situer le seuil à $1,2-1,4 \mathrm{~J} / \mathrm{cm}^{2}$, également en bon accord avec les prévisions des modèles théoriques.

\subsection{INTRODUCTION DE DÉFAUTS. - La dépendance} de la capacité des diodes avec la fréquence du signal de mesure indique la présence, dans les zones irradiées, de niveaux pièges profonds. L'évolution de la capacité avec la tension appliquée montre par ailleurs que l'irradiation introduit des donneurs électriquement 
actifs, de sorte que la concentration de niveaux ionisés varie au voisinage de la surface. Nous reviendrons plus loin sur l'énergie et la nature des différents défauts déjà repérés par quelques auteurs. La présence de dommages dans la zone de charge d'espace peut expliquer la diminution de la tension de claquage, l'augmentation du courant inverse des diodes [20], et celui de $n$, lié à un taux de recombinaison élevé ou à une probabilité non négligeable de transport du courant par effet tunnel. Deux facteurs sont susceptibles d'affecter la barrière de potentiel : une dégradation de la surface (par l'intermédiaire des états de surface ou par le changement des propriétés de la couche d'oxyde natif), ou encore une modification de la courbure des bandes en surface, liée au déplacement du niveau de Fermi par suite de la présence de niveaux ionisés. Bien que les processus mis en jeu soient probablement différents, on peut noter que le bombardement de la surface de silicium par des ions peu énergétiques ou le dépôt d'une électrode par pulvérisation cathodique conduisent à des résultats analogues [21].

3.3 Profil DE Distribution des déFAUTS. - On a essayé de déterminer le profil de distribution des défauts introduits dans le cas d'une irradiation à $1,45 \mathrm{~J} / \mathrm{cm}^{2}$ en exploitant les mesures de capacité effectuées en fonction de la profondeur d'attaque du silicium. A cet effet, on a cherché à reproduire par le calcul les courbes capacité/épaisseur décapée de la figure 5, en résolvant l'équation de Poisson compte tenu d'une distribution de donneurs $N(y)$ se superposant à la concentration d'impuretés du matériau de base, $N_{\mathrm{D}}$. Un bon lissage de ces courbes a été obtenu en utilisant une distribution de la forme :

$$
N(y)=N_{\mathrm{T}} \exp (-\alpha y) \text {. }
$$

L'équation de Poisson qui permet de calculer l'épaisseur de la zone de charge d'espace $w$, d'où l'on déduit la capacité est alors :

$$
\begin{aligned}
q N_{\mathrm{D}} w^{2} /\left(2 \varepsilon_{\mathrm{S}}\right)- & N_{\mathrm{T}}\left(q / \varepsilon_{\mathrm{S}} \alpha^{2}\right)(1+\alpha w) \times \\
& \times \exp [-\alpha(x+w)]+\left(q N_{\mathrm{T}} / \varepsilon_{\mathrm{S}} \alpha^{2}\right) \\
& \times \exp (-\alpha x)+V_{0}+V=0
\end{aligned}
$$

dans lequelle $x$ représente l'épaisseur de silicium décapée, $V_{0}$ la tension de diffusion, $V$ la tension appliquée, $q$ la charge de l'électron et $\varepsilon_{\mathrm{S}}$ la constante diélectrique. Pour une tension de polarisation inverse de un volt, l'ajustement des courbes est obtenu en attribuant aux paramètres $N_{\mathrm{T}}$ et $\alpha$ les valeurs $N_{\mathrm{T}}=5,5 \times$ $10^{17} / \mathrm{cm}^{3}$ et $\alpha=2,3 \times 10^{-2} \mathrm{~nm}^{-1}$. Pour une tension de polarisation de $-0,5 \mathrm{~V}$, l'accord entre les courbes calculées conduit à la valeur $N_{\mathrm{T}}=3,0 \times 10^{17} / \mathrm{cm}^{3}$, $\alpha$ demeurant identique ; toutefois la forte indétermination qui règne sur la valeur de $V_{0}$ ne permet pas d'atteindre un lissage aussi bon que dans le premier cas.

Ce type de distribution suggérant, comme cela a déjà été remarqué [17], un processus de diffusion, on a pour comparaison pris en considération un profil de distribution de la forme :

$$
N(y)=N_{\mathrm{T}} \operatorname{erfc}(y / 2 \sqrt{D t})
$$

qui, entre 0 et $100 \mathrm{~nm}$, est très proche de la courbe exponentielle si l'on prend pour $D t$ la valeur $D t=$ $1,7 \times 10^{-11} \mathrm{~cm}^{2}$. En se plaçant dans l'hypothèse du modèle thermique de l'interaction laser-silicium, on peut estimer le temps de fusion $t$ à $200 \mathrm{~ns}$ et le coefficient de diffusion $D$ à $8,5 \times 10^{-5} \mathrm{~cm}^{2} / \mathrm{s}$, très proche par conséquent des valeurs généralement trouvées pour la diffusion des impuretés dans le silicium en phase liquide [22].

La décroissance très rapide de ces distributions ne permet pas de les déduire à partir de l'expérience pour des profondeurs supérieures à $100-120 \mathrm{~nm}$; toutefois, leur extrapolation jusqu'à $300-400 \mathrm{~nm}$ suggère que des densités notables de défauts (comparables à la concentration en impuretés ionisées dans le matériau de base), pourraient s'étendre jusqu'à $200-300 \mathrm{~nm}$, soit à des profondeurs qui correspondent à l'épaisseur de la couche de silicium fondue lors d'une irradiation à $1,45 \mathrm{~J} / \mathrm{cm}^{2}$ [19].

La modification du dopage entraînant un changement de la position du niveau de Fermi par rapport à la bande de conduction, à la surface l'abaissement de la bande de conduction par rapport au volume, pour du silicium de $5 \Omega . \mathrm{cm}$, atteint $0,16 \mathrm{eV}$ : on peut donc attendre, pour un contact d'or, une barrière de potentiel d'environ $0,64 \mathrm{eV}$, valeur un peu plus élevée que celle associée au courant de saturation, soit $0,55 \mathrm{eV}$. Cette différence pourrait provenir soit d'une modification des propriétés de la couche d'oxyde natif lors de l'irradiation [14] ou encore du mode de détermination employé, fondé sur un transport du courant par effet purement thermoionique, alors que la concentration élevée de donneurs en surface permet d'envisager un effet tunnel.

3.4 RECUIT THERMIQUE ET NATURE DES DÉFAUTS. D'après les mesures de capacité reportées sur la figure 6, un recuit thermique à $600^{\circ} \mathrm{C}$ est nécessaire pour faire disparaître les défauts introduits lors de l'irradiation. Toutefois, il semble que même un recuit à $700^{\circ} \mathrm{C}$ ne permette pas d'obtenir des caractéristiques $I-V$ semblables à celles d'un échantillon témoin. Pour une irradiation par laser provoquant la fusion brève du silicium, deux niveaux d'énergie ont été identifiés dans la bande interdite [17] : le premier situé à $E_{\mathrm{c}}-0,19 \mathrm{eV}$ se recuit à $550^{\circ} \mathrm{C}$, le second placé à $E_{\mathrm{c}}-0,33 \mathrm{eV}$ disparaît après chauffage à $650^{\circ} \mathrm{C}$. (La température de recuit peut éventuellement être abaissée à $200^{\circ} \mathrm{C}$ par interaction des défauts avec l'hydrogène atomique [23].) Cependant des défauts ponctuels pourraient subsister jusqu'à $700^{\circ} \mathrm{C}$ et au-delà. La nature de ces défauts demeure encore mal définie, la majeure partie des travaux concernant le recuit impulsionnel du silicium ayant trait à des couches implantées. 
L'hypothèse la plus généralement avancée fait état de l'incorporation et de la diffusion d'oxygène, ou de complexes associés à l'oxygène, à caractère donneur, dans le silicium fondu. Toutefois, l'analyse de la concentration d'oxygène dans le silicium recouvert d'une couche d'oxyde natif de quelques nanomètres d'épaisseur, montre des différences notables selon que l'irradiation par laser a été effectuée sous ultra-vide [13] ou sous atmosphère d'oxygène [14] : seul l'oxygène provenant de l'atmosphère ambiante semble pénétrer dans le matériau, l'influence de l'oxyde ne se manifestant que par la modification de la densité d'états de surface et de la charge qu'il contient. On peut noter que l'irradiation en continu par un faisceau d'électrons ou un laser qui ne provoquent pas la fusion du silicium entraînent, entre autre, l'apparition du niveau piège situé à $E_{\mathrm{c}}-0,19 \mathrm{eV}[24,25]$, dont la distribution à partir de la surface est exponentielle. Son attribution à une origine microscopique encore indéterminée [25] ou à une association faisant intervenir l'oxygène [24] demeure également controversée. Le niveau piège situé à $E_{\mathrm{c}}-0,33 \mathrm{eV}$, seulement mis en évidence lorsque l'interaction laser-silicium implique la présence d'une phase liquide, pourrait être dû à des dislocations [17, 25] ou à la formation de complexes $\mathrm{V}-\mathrm{C}_{\mathrm{s}}-\mathrm{O}_{\mathrm{I}}$ impliquant du carbone substitutionnel et de l'oxygène interstitiel.

Les travaux les plus récents [26] font état de l'apparition après irradiation à $0,9 \mathrm{~J} / \mathrm{cm}^{2}$ de trois niveaux d'énergie situés respectivement à $E_{\mathrm{c}}-0,25, E_{\mathrm{c}}-0,32$ et $E_{\mathrm{c}}-0,49 \mathrm{eV}$, identiques aux niveaux produits par une trempe rapide du silicium. Ils pourraient être attribués à des lacunes créées à haute température et piégées lors du refroidissement, l'atmosphère ambiante lors du traitement ne jouant aucun rôle. Ces défauts, qui disparaissent après un recuit thermique à $600^{\circ} \mathrm{C}$, ne correspondent cependant pas tout à fait aux déterminations antérieures sur le silicium de type $\mathbf{N}$ que nous avons rappelées plus haut.

4. Conclusion. - L'irradiation de silicium vierge par l'impulsion d'un laser à rubis provoque la création d'une importante densité de défauts, distribués dans la zone fondue pendant l'interaction. Il en résulte une profonde modification des propriétés du matériau, qui peut se répercuter sur le fonctionnement des dispositifs. Les caractéristiques des diodes Schottky sont par exemple très fortement affectées par ce traitement lorsque l'énergie des impulsions dépasse le seuil de fusion du silicium. De plus, un recuit thermique ne permet qu'en partie de supprimer les effets néfastes de l'irradiation. Après avoir étudié le comportement de diodes $\mathrm{Au}-\mathrm{Si} \mathrm{N}$, on a effectué quelques expériences préliminaires sur du silicium de type $P$ : on a également pu constater une détérioration des diodes, Al-Si P lorsque l'énergie augmente, moins prononcée cependant que dans le cas du silicium N. Des expériences sont en cours afin d'essayer de préciser la nature des défauts introduits et le rôle que l'oxygène pourrait éventuellement jouer dans leur création, soit par diffusion à partir de l'air ambiant, soit par ségrégation vers la surface.

\section{Bibliographie}

[1] Levine, L. P., Ready, J. F., Bernal, E., J. Appl. Phys. 38 (1967) 331.

[2] Bedair, S., Smith, H. P., J. Appl. Phys. 40 (1969) 4776.

[3] RapPaRINI, R., Vacuum 21 (1971) 385.

[4] Wautelet, M., Surf. Sci. 95 (1980) 299.

[5] Zehner, D. M., White, C. W., OWnby, G. W., Appl. Phys. Lett. 36 (1980) 56.

[6] Zehner, D. M., White, C. W., Ownby, G. W., Surf. Sci. 92 (1980) L-67.

[7] Cowan, P. L., Golovchenko, J. A., J. Vac. Sci. Technol. 17 (1980) 1197.

[8] Mc Kinley, A., Parke, A. W., Hughes, G. J., Fryar, J., Williams, R. H., J. Phys. D. Appl. Phys. 13 (1980) L-193.

[9] Bermudez, V. M., J. Vac. Sci. Technol. 20 (1982) 51.

[10] Mc Kinley, A., Williams, R. H., Parke, A., SRIVASTANa, G. P., Vacuum 31 (1981) 549.

[11] Hoh, K., Koyama, H., UdA, K., MiURA, Y., Japan J. Appl. Phys. 19 (1980) L-375.

[12] Garulli, A., Servidori, M., Vecchi, I., J. Phys. D : Appl. Phys. 13 (1980) L-199.

[13] WestendorP, H., Zhong-Lie Wang, Saris, F., A paraître dans Proc. Material Research Society, Boston 1981.

[14] Deshmukh, V. G. I., Webber, H. C., Mc Caughan, D. V., Appl. Phys. Lett. 39 (1981) 25.

[15] Ready, J. F., J. Appl. Phys. 36 (1965) 462.
[16] Bhattacharya, P. K., Phys. Status Solidi (a) 59 (1980) 827.

[17] Kimmerling, L. C., Benton, J. L., Laser and Electron Beam Processing of Materials C. W. White, P. S. Peercy, eds (Academic Press, New York) 1979, p. 385 .

[18] Mooney, P. M., Young, R. T., Karins, J., LeE, Y. H., Corbett, J. W., Phys. Status Solidi (a) 48 (1978) K31.

[19] Bell, R. O., Toulemonde, M., Siffert, P., Appl. Phys. 19 (1979) 313.

[20] Milnes, A. G., Deep Impurities in Semiconductors (J. Wiley and Sons eds.) 1973, p. 187.

[21] Berg, S., Anderson, L. P., Norstrom, M., Grusell, E., Vacuum 27 (1977) 189.

[22] Kodera, H., Japan J. Appl. Phys. 2 (1963) 212.

[23] Benton, J. L., Doherty, C. J., Ferris, S. D., Flamm, D. L., Kimmerling, L. C., Leamy, H. J., Appl. Phys. Lett. 36 (1980) 670.

[24] Johnson, N. M., Regolini, J. L., Bartelink, D. J., Gibbons, J. F., Ratnakumar, K. N., Appl. Phys. Lett. 36 (1980) 425.

[25] Chantre, A., Kechouane, M., Bois, D., A paraître Proc. Material Research Society, Boston 1981.

[26] Fan, Z. K., Ho, V. D., Sugano, T., Appl. Phys. Lett. 40 (1982) 418 